\title{
Threshold effects in positron scattering from isoelectronic series of atoms and molecules
}

\author{
J. R. Machacek, ${ }^{1}$ S. J. Buckman, ${ }^{1,2}$ and J. P. Sullivan ${ }^{1}$ \\ ${ }^{1}$ ARC Centre for Antimatter-Matter Studies, Research School of Physics and Engineering, \\ Australian National University, Canberra 0200, Australia \\ ${ }^{2}$ Institute of Mathematical Sciences, University of Malaya, Kuala Lumpur, Malaysia
}

(Received 4 August 2014; published 9 October 2014)

\begin{abstract}
The observation of features, often referred to as "Wigner cusps" in a scattering cross section at the opening of a new scattering channel has been widely reported in electron scattering, and has been attributed to strong coupling between the two scattering channels. Recently a series of cusps were observed in positron scattering from the noble gases [A. C. L. Jones et al., Phys. Rev. Lett. 105, 073201 (2010)]. In this case, the strong opening of the positronium formation cross section resulted in a cusplike feature, or bump, in the total elastic scattering cross section. The positronium formation channel is typically the largest partial scattering cross section in the region of the electronic excitation and ionization thresholds in most atoms and molecules, and it is plausible that similar features may exist in other atomic and molecular species. We have undertaken measurements of the isoelectronic targets of helium $-\mathrm{H}_{2}$, and neon $-\mathrm{H}_{2} \mathrm{O}, \mathrm{NH}_{3}$, and $\mathrm{CH}_{4}$, in an effort to better understand the mechanisms leading to these threshold features.
\end{abstract}

DOI: 10.1103/PhysRevA.90.042703

PACS number(s): 34.80.Uv, 34.80.Lx, 34.80.Gs

\section{INTRODUCTION}

There are many examples of channel coupling in the scattering of bosons and leptons and, recently, strong evidence of channel coupling has been observed in antimatter-matter collisions [1,2]. Various forms of channel coupling can manifest in scattering cross sections. Wigner cusps [3], first articulated in the context of nuclear scattering, represent a classic example of such channel coupling. They have also been observed in low-energy electron scattering from atoms and molecules [4-6]. Another, slightly different but very common, example of channel coupling occurs in electron scattering from both atoms and molecules and involves the formation of transient negative ions, or resonances. These resonant states usually involve scattering in a single, well-defined partial wave, and interference between this partial wave and the background, nonresonant scattering waves, results in Fano-Buetler profiles in the (differential) scattering cross sections; these profiles contain considerable information about the processes involved.

Traditional studies in both nuclear (e.g., [3]) and atomic physics $[4,5]$ on Wigner cusps considered the effect on the elastic scattering channel at the energy where a new inelastic scattering channel became open. There are several key studies involving low-energy electron scattering where the opening of an inelastic scattering channels causes a cusp or "step" in the elastic scattering cross section. Excellent examples are provided in the work of Cvejanović et al. [4] who studied elastic scattering from neutral helium in the region of the $n=2$ excitation thresholds, and Eyb and Hofmann [5] who measured elastic scattering in sodium in the region of the $3{ }^{2} P$ resonance level excitation thresholds. In the latter case in particular, the features that were observed in the elastic differential cross sections at certain scattering angles were of considerable magnitude relative to the background elastic scattering cross section. The $3^{2} P$ resonance transitions in sodium account for more than $98 \%$ of the total optical oscillator strength for the atom, and the near-threshold electron excitation cross sections for these states are extremely large. Such strong, near-threshold excitation cross sections are believed to have a considerable bearing on the existence and relative strength of any cusp effects that are seen in the elastic channel.

Recently, direct observation of channel coupling effects in positron scattering by the noble gases has been revealed through the observation of threshold features, in the elastic scattering channel, at energies coinciding with the opening of the positronium (Ps) formation channel [1,2]. These works also contain summaries of previous work in the field of positron scattering, where such cusps had been the subject of speculation for many years (e.g., [7-11]). In the most recent work [2], no obvious trend was found in the observed structures across the rare gas atoms, particularly when attempting to relate the strength of the effect to atomic parameters such as dipole polarizability.

The Ps formation threshold is lower in energy than the threshold energy for the first electronic excited state for all of the noble gas atoms. If direct annihilation is neglected, and this is a vanishingly small cross section at these energies, then the only open channel below, and at, the Ps formation threshold in each of the rare gases, is the elastic scattering channel. On the other hand, other atomic targets, and all molecules, may have one or more (or many) other inelastic channels open at the energy corresponding to the Ps formation threshold. In particular, molecules have additional degrees of freedom, rotations, and vibrations and, in some cases, these extra degrees of freedom provide for additional scattering channels. Many of these rotational and vibrational scattering channels are open at the energy of the Ps formation threshold. A key question that can be raised in the case of both atoms and molecules is whether the presence of a high density of open scattering channels, over which the channel coupling effects may be shared, has an effect on the presence or absence of these threshold cusps and features. In the present work we provide some comparisons between positron scattering systems where threshold cusps have been clearly observed ( $\mathrm{He}$ and $\mathrm{Ne}$ ) and some isoelectronic molecular counterparts to these atoms - in the case of He we investigate the $\mathrm{H}_{2}$ molecule, 


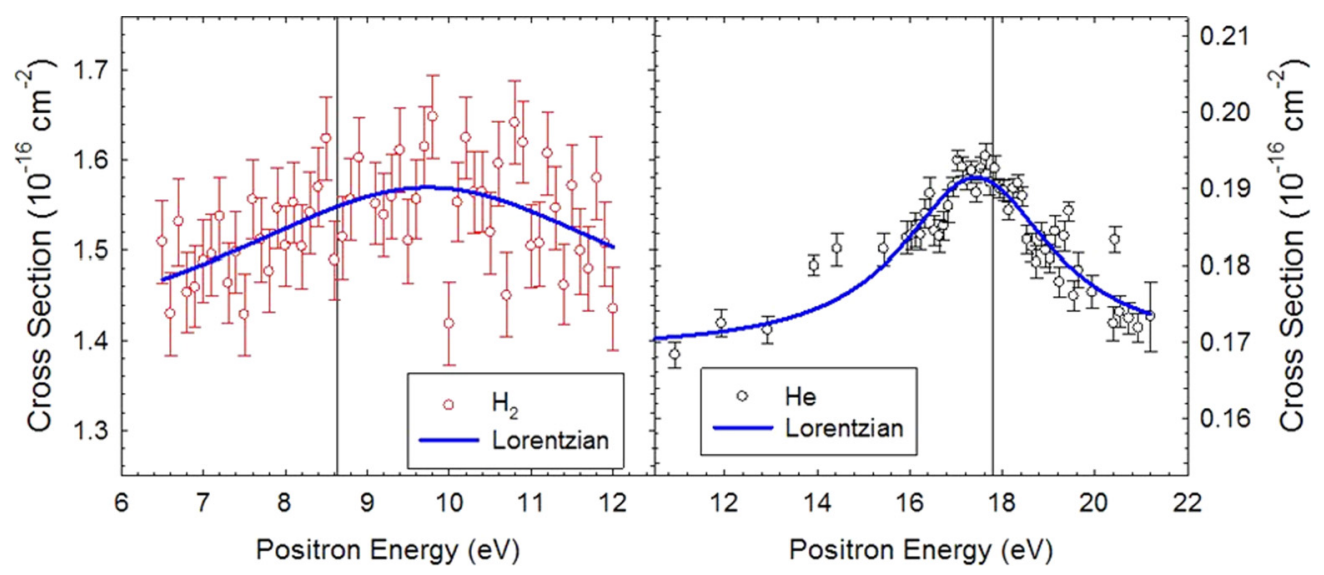

FIG. 1. (Color online) Comparison of elastic cross sections for He (right panel) and $\mathrm{H}_{2}$ (left panel) in the vicinity of the Ps formation threshold, which is indicated by the solid vertical line in each panel. The solid lines in both panels are fits to the experimental data consisting of a linear background and a Lorentzian profile. The helium data are from Jones et al. [2].

and for $\mathrm{Ne}$ we compare with $\mathrm{CH}_{4}, \mathrm{H}_{2} \mathrm{O}$, and $\mathrm{NH}_{3}$ molecules. The main rationale for these studies is to investigate the role that atomic and molecular structure and properties might play in the formation, or otherwise, of these threshold features in systems that have identical numbers of electrons.

\section{EXPERIMENTAL CONSIDERATIONS}

The positron scattering experiments were undertaken on the low-energy positron beamline at the Australian National University, which has been described in detail in a previous publication [12]. A ${ }^{22} \mathrm{Na}$ positron source with an activity of approximately $30 \mathrm{mCi}$ was used as a source of energetic positrons. These positrons were moderated to an energy of approximately $2 \mathrm{eV}$ by using a solid krypton moderator; the krypton was sprayed as gas onto the positron source capsule which was held at a temperature of $\sim 20 \mathrm{~K}$. The moderated positrons were magnetically guided from the source region at a low energy, $\sim 8 \mathrm{eV}$, and injected into a Surko-style buffer-gas trap where they were collisionally cooled and trapped via interactions with $\mathrm{N}_{2}$ and $\mathrm{CF}_{4}$ gases. The resulting cold positron cloud was then extracted from the trap in a pulsed beam at a rate of $50 \mathrm{~Hz}[\Delta E \sim 50 \mathrm{meV}$ full width at half maximum (FWHM)], and transported to a scattering cell containing the target gas of interest. Scattered and unscattered positrons were energy analyzed using a cylindrically symmetric, retarding potential analyzer (RPA) prior to being detected with a multichannel plate (MCP). The energy loss of the scattered positrons, measured using the RPA, was used to determine what type of scattering the positron underwent. Ps formation was determined by measuring the difference in the number of positrons traversing the gas cell below the Ps formation threshold and at a scattering energy above the Ps formation threshold. The uncertainty in the absolute energy of the beam, measured using the RPA, is estimated to be $\pm 50 \mathrm{meV}$. This can also be verified by the observation of the threshold of the Ps formation cross section.

In the present series of measurements, three cross sections are measured effectively at the same time-the grand total cross section, the total (quasi-)elastic cross section, and the
Ps formation cross section. The techniques that are used for these measurements have been discussed in detail previously [12-14], so we shall not repeat them here. While absolute values for these measurements are obtained, that is not the main goal of this study, which is to search for structures in the total elastic scattering cross section in the proximity of the Ps formation threshold.

\section{EXPERIMENTAL RESULTS}

Our first experimental comparison of cusp behavior in isoelectronic atoms and molecules involves the two-electron systems, helium and molecular hydrogen, and this comparison is provided in Fig. 1. The right-hand panel shows the behavior of the total elastic cross section in helium in the vicinity of the Ps threshold (18.78 eV) and the left-hand panel is a similar plot for the $\mathrm{H}_{2}$ molecule near the Ps threshold which occurs at $8.63 \mathrm{eV}$. The helium data are taken from the work of Jones et al. [2]. A relatively broad, cusplike feature is clearly evident in the He cross section, and it is centered only slightly above the Ps formation threshold. On the other hand, in the case of $\mathrm{H}_{2}$, it is possible that a very broad structure is seen in the elastic cross section, in that the cross section does decrease after the Ps threshold, but the "peak" in this feature lies several electron volts above the Ps threshold, and is therefore probably not directly associated with the opening of the Ps channel in any way.

In Fig. 2, we show measurements of elastic scattering and Ps formation near the Ps threshold for the ten-electron systems, $\mathrm{Ne}, \mathrm{H}_{2} \mathrm{O}, \mathrm{NH}_{3}$, and $\mathrm{CH}_{4}$. The current results for $\mathrm{Ne}$, shown in the top panel of Fig. 2, confirm the magnitude and shape of the cusp that was previously observed by Jones et al. [2]. Of all the noble gases, Ne has the weakest cusp in the elastic channel, with the feature representing only about $4 \%$ of the total scattering cross section at the Ps formation threshold. The second panel of Fig. 2 shows results for elastic scattering from water in the energy region of the Ps threshold $(5.82 \mathrm{eV})$. The present data agree with that taken previously for water [15], where we did not detect a feature in the elastic cross section at or near the Ps threshold. Once again in the present 

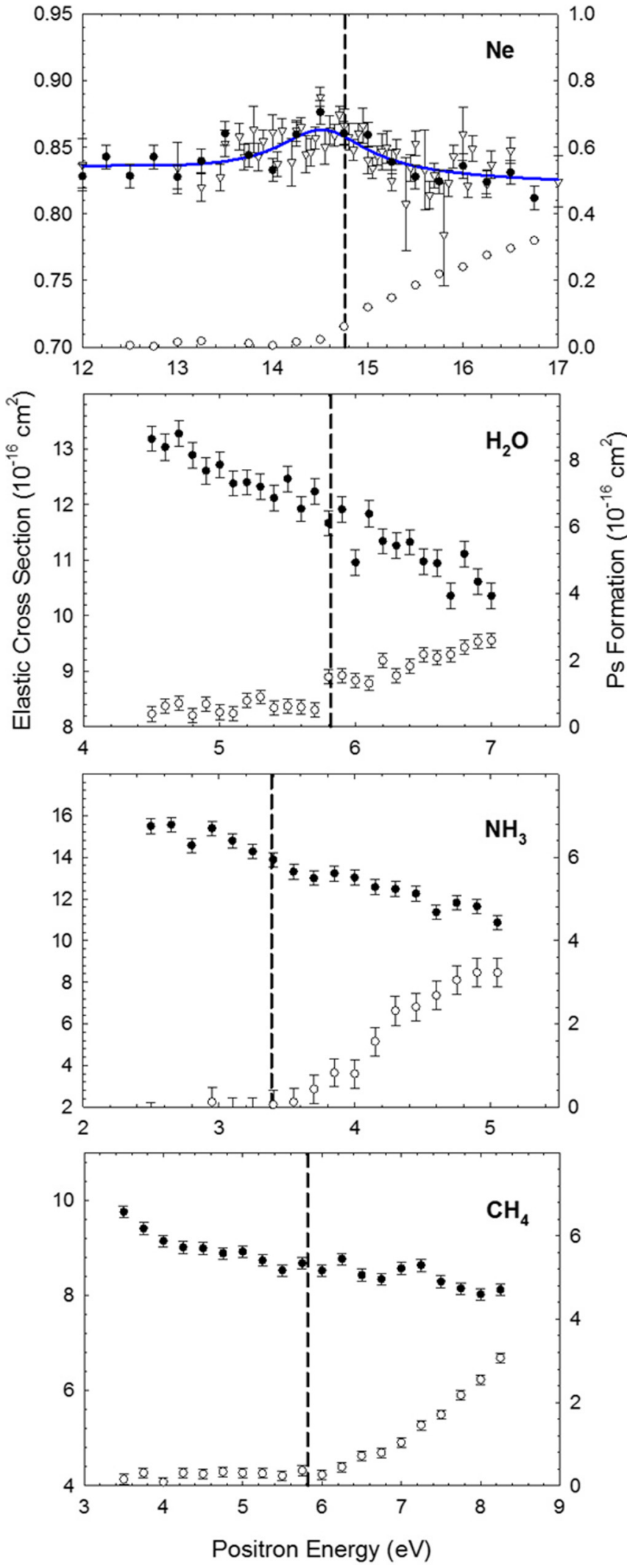

FIG. 2. (Color online) Elastic scattering and positronium (Ps) formation cross sections for positron scattering from (top panel) neon $(\mathrm{Ne})$, (second panel) water $\left(\mathrm{H}_{2} \mathrm{O}\right)$, (third panel) ammonia $\left(\mathrm{NH}_{3}\right)$, and (bottom panel) methane $\left(\mathrm{CH}_{4}\right)$, in the energy region about their Ps formation thresholds. data there is no evidence of any structure, or cusp feature, at or near the Ps threshold. The third panel shows elastic cross section measurements for ammonia $\left(\mathrm{NH}_{3}\right)$ at energies near the Ps threshold for this molecule $(3.39 \mathrm{eV})$. The grand total cross section results of Sueoka et al. [16] are slightly lower than those reported here and have not been included in the interests of clarity. Once again there is no compelling evidence for the presence of any threshold-related feature in this cross section. The final panel of Fig. 2 shows the elastic cross section for methane $\left(\mathrm{CH}_{4}\right)$ in the vicinity of the Ps threshold $(5.82 \mathrm{eV})$. While there are previous measurements of positron scattering from $\mathrm{CH}_{4}$ of the grand total scattering cross section from several authors [16-21], more work needs to be done on measuring the total elastic cross section. Yet again, we see no evidence of structure in the measured cross section near the Ps threshold.

\section{DISCUSSION}

Prior to a discussion of the experimental results it is interesting to note the properties (dipole moments, polarizabilities, vibrational energy levels, etc.) of the four molecules in question in this study. These are summarized in Table I, but do not include rotational threshold energies as there are many in the heavier molecules; all lie below $50 \mathrm{meV}$. As we shall explore, these parameters likely hold the key to explaining the lack of threshold structures in these molecules.

As we have seen, no threshold features (Wigner cusps or otherwise) have been observed for positron scattering from a range of molecules that are isoelectronic to either the $\mathrm{He}$ or $\mathrm{Ne}$ atoms. In the case of $\mathrm{H}_{2}$ a broad, above-threshold feature is observed, centered some $2 \mathrm{eV}$ above the Ps threshold, but this does not possess any of the characteristics observed by Jones et al. [2] in their study of the rare gases, and in particular the He atom.

At this point we recall that for all of the noble gases, at the energy at which the Ps formation channel opens, there is only one other scattering channel which is open-that for elastic scattering. Thus the flux that is lost from the incident scattering channel due to near-threshold Ps formation is only coupled into the elastic scattering channel. In all of the rare gases the next scattering channel, electronic excitation of the lowest-lying state(s), does not open until several eV above the Ps formation threshold (neglecting direct annihilation, which is extremely small at these energies). Thus in the region between Ps threshold and the first electronic excited state threshold, known as the Ore gap, the only "open" channels are elastic scattering and Ps formation.

In contrast, for each of the molecular targets that are considered here, there are additional degrees of freedom, in particular vibrational and rotational motion, which lead to many additional scattering channels being open in the region of the Ps threshold. Molecules can also possess a dipole moment which can strongly enhance scattering channels and, in particular, lead to very large cross sections at low energies and/or small scattering angles [22]; see Table I for the relevant parameters.

While it is evident that for the four molecules considered here there are many open scattering channels (when including overtones and combination modes) at the energy where the Ps 
TABLE I. Some atomic and molecular properties of the target species considered here [28].

\begin{tabular}{|c|c|c|c|c|c|c|}
\hline & $\mathrm{He}$ & $\mathrm{H}_{2}$ & $\mathrm{Ne}$ & $\mathrm{CH}_{4}$ & $\mathrm{H}_{2} \mathrm{O}$ & $\mathrm{NH}_{3}$ \\
\hline Ps threshold (eV) & 17.78 & 8.63 & 14.76 & 5.82 & 5.82 & 3.39 \\
\hline Dipole polarizability $\left(a_{0}^{3}\right)$ & 1.38 & 6.74 & 24.56 & 17.61 & 9.78 & 14.56 \\
\hline Dipole moment (D) & 0 & 0 & 0 & 0 & 1.85 & 1.42 \\
\hline Vibrational threshold (eV) & & 0.545 & & 0.162 & 0.198 & 0.118 \\
\hline Electronic excitation threshold $(\mathrm{eV})$ & 20.6 & 11.8 & 16.6 & 8.61 & 7.14 & 5.67 \\
\hline
\end{tabular}

threshold is reached, what is not well known, in most cases, are the magnitudes of the cross sections for these scattering channels. In the case of the isoelectronic series of $\mathrm{Ne}$, the first electronic excitation is above the Ps formation threshold for all three molecules; see Table I. However, each molecule has a number of fundamental modes, Table II, which open at energies of, typically, hundreds of meV. Fortunately, the cross section for positron-impact vibrational excitation has been measured for methane by Sullivan et al. [22] and compared to electron-impact vibrational excitation. This comparison shows good agreement given the limitations of the available electron scattering data. Therefore, for the purposes of this discussion, we will assume that electron-impact vibrational excitation can be used to estimate the magnitude of the vibrational cross section about the Ps formation threshold.

\section{A. Hydrogen}

In the case of molecular hydrogen, the $v_{1}$ vibrational excitation by positron impact has been measured [23]. This measurement extends from threshold to $4 \mathrm{eV}$, but indicates that the cross section for positron-impact vibrational excitation is slowly decreasing as a function of energy. It is reasonable to assume, from this measurement, that the magnitude of vibrational excitations in $\mathrm{H}_{2}$ is $<5 \%$ of the total scattering cross section. This nonzero vibrational channel may be a sufficient condition to suppress a Wigner cusp below our current ability to measure it considering that the cusp measured in helium was $11 \%$ [2].

\section{B. Water}

Water has three normal vibrational modes listed in Table II. An attempt at measuring these modes, in the course of this

TABLE II. Vibrational mode energies and symmetries [28].

\begin{tabular}{lcccc}
\hline \hline Target & Mode & $\mathrm{E}_{v}(\mathrm{meV})$ & Symmetry & Sym. Species \\
\hline $\mathrm{H}_{2} \mathrm{O}$ & $v_{1}$ & 453.4 & $C_{2 v}$ & $a_{1}$ Sym. str. \\
& $v_{2}$ & 197.8 & & $a_{1}$ Bend \\
& $v_{3}$ & 465.7 & & $b_{2}$ Anti str. \\
$\mathrm{CH}_{4}$ & $v_{1}$ & 361.7 & $T_{d}$ & $a_{1}$ Sym. str. \\
& $v_{2}$ & 190.2 & & $e$ Bend \\
& $v_{3}$ & 374.3 & & $f_{2}$ Deg. str. \\
& $v_{4}$ & 161.9 & & $f_{2}$ Deg. deform \\
$\mathrm{NH}_{3}$ & $v_{1}$ & 413.7 & $C_{3 v}$ & $a_{1}$ Sym. str. \\
& $v_{2}$ & 117.8 & & $a_{1}$ Sym. deform \\
& $v_{3}$ & 427.0 & & $e$ Deg. str. \\
& $v_{4}$ & 201.7 & & $e$ Deg. deform \\
\hline \hline
\end{tabular}

study, was unable to completely separate the second vibrational mode from elastic scattering, but preliminary results were not inconsistent in magnitude with the electron scattering data of Khakoo et al. and El-Zein et al. [24,25]. Using these electron data we can estimate the magnitude of the total $\left(v_{1}+v_{2}+v_{3}\right)$ vibrational cross section at the opening of the Ps formation channel is nearly $1 \AA^{2}$. This is nearly $10 \%$ of the total scattering cross section at the Ps formation threshold, while the magnitude of the cusp in neon was approximately $4 \%$ [2]. Thus, it is possible that the existence of these many vibrational scattering channels with nonzero magnitude may not provide a favorable configuration for a Wigner cusp in the elastic scattering cross section.

\section{Ammonia}

Ammonia has four normal vibrational modes listed in Table II, none of which have been explored by positron impact. Results for vibrational excitation via electron impact are sparse for ammonia, but some differential electron scattering data were reported by Gulley et al. [26] at energies near the Ps formation threshold energy. Integrating the differential cross section for the first and third vibrational modes nearest the Ps threshold yields a total vibrational cross section value of $0.58 \AA^{2}$. This is $\sim 4 \%$ percent of the grand total scattering cross section at the Ps formation threshold of $3.39 \mathrm{eV}$.

\section{Methane}

Methane has four normal vibrational modes. The cross section for the excitation of these modes from ground state $\mathrm{CH}_{4}$ by positron impact has been measured by Sullivan et al. [22]. It should be noted that the first and third modes are essentially degenerate, as are the second and the fourth. The finite energy resolution of $\sim 25 \mathrm{meV}$ used was insufficient to separate the normal modes completely [22], but these measurements extended from threshold to $4 \mathrm{eV}$. A modified Born approximation [27] gave good agreement for the energy dependence of the vibrational excitation which can be used to estimate the magnitude for the excitation of the $v_{1}+v_{3}$ modes to be $\sim 0.2 \AA^{2}$ and for the excitation of the $v_{2}+v_{4}$ modes $\sim 0.1 \AA^{2}$. Therefore, at the onset of Ps formation we estimate that the total $\left(v_{1}+v_{2}+v_{3}+v_{4}\right)$ cross section for vibrational excitation is $0.3 \AA^{2}$ with four channels open. Measurements of the elastic scattering cross section in the region about the Ps formation threshold are shown in Fig. 2. No cusp feature is observed about the Ps formation threshold. At this energy the vibrational excitation cross section has an estimated magnitude of $\sim 4 \%$ of the total scattering cross section. 


\section{CONCLUSION}

The present investigation represents detailed measurements searching for channel coupling in the form of Wigner cusps in positron scattering from molecules $\mathrm{H}_{2}, \mathrm{H}_{2} \mathrm{O}, \mathrm{NH}_{3}$, and $\mathrm{CH}_{4}$. The cusp observed in neon and the total vibrational excitation of these molecules are of a similar magnitude compared to the total scattering cross section. The null measurement of a cusp feature in these molecules is likely due to nonzero vibrational excitations at the Ps formation threshold suppressing the magnitude of a cusp to below that detectible by the current experimental setup. The presence of many overtone modes, even if the excitation is very small, will only enhance this suppression. A further detailed experimental study of positron scattering from molecules, with and without dipole moments, and, with different symmetries (from linear to spherical), may provide further insight. In addition, exploration of the elastic scattering channel about the threshold(s) for vibrational excitation by positron impact has not been explored in detail, to date, but provides an additional configuration favorable for Wigner cusps. Theoretical work in calculating the magnitude and shape of Wigner cusps would be of great utility to a future exploration to guide the experimental requirements. It is possible that cusps are present, but smaller in magnitude than the current set of experiments is able to measure. This limits the utility of these measurements in determining a trend in cusp formation in positron scattering from molecules which might provide physical insight into this coupling phenomena. As no trend was found in the cusp results for the noble gases [2], a more thorough theoretical and experimental investigation of these cusps and their formation mechanism is needed.

\section{ACKNOWLEDGMENTS}

The authors would like to acknowledge the Australian Research Council's Centre of Excellence Program for providing funding. We would also like to thank the technical staff, Graeme Cornish, Stephen Battisson, Ross Tranter, and Ron Cruikshank, for their frequent assistance and technical advice. Finally, we would like to thank Dr. Simon Armitage for assistance with growing $\mathrm{Kr}$ moderators. S.J.B. warmly acknowledges the University of Malaya for their support as a Distinguished Visiting Professor.
[1] P. G. Coleman, N. Cheesman, and E. R. Lowry, Phys. Rev. Lett. 102, 173201 (2009).

[2] A. C. L. Jones, P. Caradonna, C. Makochekanwa, D. S. Slaughter, R. P. McEachran, J. R. Machacek, J. P. Sullivan, and S. J. Buckman, Phys. Rev. Lett. 105, 073201 (2010).

[3] E. P. Wigner, Phys. Rev. 73, 1002 (1948).

[4] S. Cvejanović, J. Comer, and F. H. Read, J. Phys. B 7, 468 (1974).

[5] M. Eyb and H. Hofmann, J. Phys. B 8, 1095 (1975).

[6] M. A. Khakoo, S. Wang, R. Laher, P. V. Johnson, C. P. Malone, and I. Kanik, J. Phys. B 40, F167 (2007).

[7] P. G. Coleman, K. A. Johnston, A. M. G. Cox, A. Goodyear, and M. Charlton, J. Phys. B 25, L585 (1992).

[8] G. Laricchia, J. Moxom, and M. Charlton, Phys. Rev. Lett. 70, 3229 (1993).

[9] J. Moxom, G. Laricchia, M. Charlton, A. Kovar, and W. E. Meyerhof, Phys. Rev. A 50, 3129 (1994).

[10] W. E. Meyerhof, G. Laricchia, J. Moxom, J. W. Humberston, and M. S. T. Watts, Can. J. Phys. 74, 427 (1996).

[11] W. E. Meyerhof and G. Laricchia, J. Phys. B 30, 2221 (1997).

[12] L. S. Fornari, L. M. Diana, and P. G. Coleman, Phys. Rev. Lett. 51, 2276 (1983).

[13] J. P. Sullivan, A. Jones, P. Caradonna, C. Makochekanwa, and S. J. Buckman, Rev. Sci. Instrum. 79, 113105 (2008).

[14] J. R. Machacek, E. K. Anderson, C. Makochekanwa, S. J. Buckman, and J. P. Sullivan, Phys. Rev. A 88, 042715 (2013).

[15] C. Makochekanwa, A. Bankovic, W. Tattersall, A. Jones, P. Caradonna, D. S. Slaughter, K. Nixon, M. J. Brunger, Z. Petrovic, J. P. Sullivan, and S. J. Buckman, New J. Phys. 11, 103036 (2009).
[16] O. Sueoka, S. Mori, and Y. Katayama, J. Phys. B 20, 3237 (1987).

[17] A. Zecca, L. Chiari, E. Trainotti, A. Sarkar, S. d'A. Sanchez, M. H. F. Bettega, M. T. do N. Varella, M. A. P. Lima, and M. J. Brunger, Phys. Rev. A 85, 012707 (2012).

[18] M. S. Dababneh, Y. -F. Hsieh, W. E. Kauppila, C. K. Kwan, S. J. Smith, T. S. Stein, and M. N. Uddin, Phys. Rev. A 38, 1207 (1988).

[19] O. Sueoka and S. Mori, J. Phys. B 19, 4035 (1986).

[20] K. Floeder, D. Fromme, W. Raith, A. Schwab, and G. Sinapius, J. Phys. B 18, 3347 (1985).

[21] M. Charlton, T. C. Griffith, G. R. Heyland, and G. L. Wright, J. Phys. B 16, 323 (1983).

[22] J. P. Sullivan, S. J. Gilbert, J. P. Marler, L. D. Barnes, S. J. Buckman, and C. M. Surko, Nucl. Instrum. Methods Phys. Res., Sect. B 192, 3 (2002).

[23] J. P. Sullivan, S. J. Gilbert, and C. M. Surko, Phys. Rev. Lett. 86, 1494 (2001).

[24] M. A. Khakoo, C. Winstead, and V. McKoy, Phys. Rev. A 79, 052711 (2009).

[25] A. A. A. El-Zein, M. J. Brunger, and W. R. Newell, J. Phys. B 33, 5033 (2000).

[26] R. J. Gulley, M. J. Brunger, and S. J. Buckman, J. Phys. B 25, 2433 (1992).

[27] J. P. Marler, G. F. Gribakin, and C. M. Surko, Nucl. Instrum. Methods Phys. Res., Sect. B 247, 87 (2006).

[28] NIST Chemistry WebBook, NIST Standard Reference Database Number 69, edited by P. J. Linstrom and W. G. Mallard, http://webbook.nist.gov/chemistry/ 